\title{
Prediction of thermal field dynamics of mould in casting using artificial neural networks
}

\author{
Florin Susac, Valentin Tăbăcaru, Nicuşor Baroiu and Viorel Păunoiu* \\ Dunărea de Jos University of Galaţi, Department of Manufacturing Engineering, 111 Domnească \\ Street, Galaţi, Romania
}

\begin{abstract}
Manufacturing a large number of cast parts made of aluminium alloy led to an increased interest in developing and applying new control techniques of the casting process. Anyway, the difficulty in estimating some important process parameters only allowed the use of some approaches which are limited to a few geometric models. Many researchers made great efforts to find the best method for monitoring and measuring thermal field dynamics of the cast and mould during solidification and cooling of the melt alloy. Acquiring very accurate data leads to best approach for solving the heat transfer problem in casting. The paper presents the prediction of thermal field dynamics of mould in permanent mould casting using artificial neural networks and based on thermal history of the cast part and the way this thermal history influences the thermal changes of the mould. It is very important to identify the relation between the thermal fields' dynamics of both cast and mould in order to create and use a control technique of the cast solidification and cooling. The necessity of controlling the cast solidification is due to the large demand of cast parts with improved mechanical properties.
\end{abstract}

\section{Introduction}

The cast/mould interface is the most critical aspect strongly related to surface and microstructure defects. In order to make an estimation of heat mass and the way this heat crosses the cast/mould interface, the boundary conditions must be correctly defined [1].

There are no many recent approaches related to conceiving and controlling the casting process based on a neural scheme that was previously created using the thermal changes history of the cast or mould.

However, the results of any numerical simulations depend on the accuracy of input parameters and imposed boundary conditions [2].

An interesting approach of controlling the continuous casting process by using artificial neural networks (ANN) is presented by Bouchouche et al. [3]. They designed and tested an ANN closed-loop control scheme of heat transfer which may be applied only in the continuous casting process.

Manjunath et al. [4] used recurrent and back propagation neural networks for developing a systematic approach to model and optimize wear behaviour of castings. The

\footnotetext{
*Corresponding author: viorel.paunoiu@ugal.ro
} 
goal was to produce excellent wear resistance squeeze castings. They used process parameters to generate the neural model and both traditional and non-traditional optimization techniques to determine the optimum process variables corresponding to minimum wear rate.

The solidification simulations of the cast parts have a tremendous applicability both for designing the casting process and improving the cast part quality [3].

Use of lightweight components is of a great importance because of reducing the vehicles weight due to high strength-to-weight ratio [6].

Powerful computing tools, such as ANN, are now used for both the prediction of surface and microstructure defects and mechanical properties [7].

Under these circumstances, it is mandatory to give effort to carefully study the thermal field dynamics of the mould in correlation to the thermal field dynamics of the cast part.

This paper presents the prediction of thermal field dynamics of mould using ANN, in permanent mould casting of a hollow cylinder cast part made of Al-Si alloy.

\section{Experimental set-up}

The experimental set-up, as already described in previous work [8], consists in a mould composed of a core and an outer wall both made of steel.

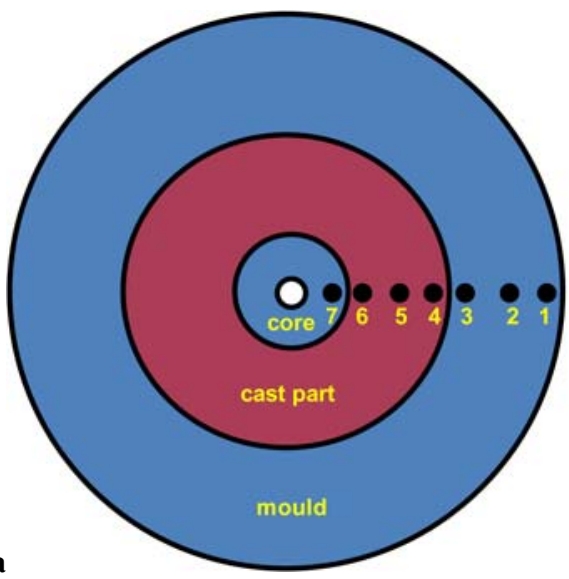

b.

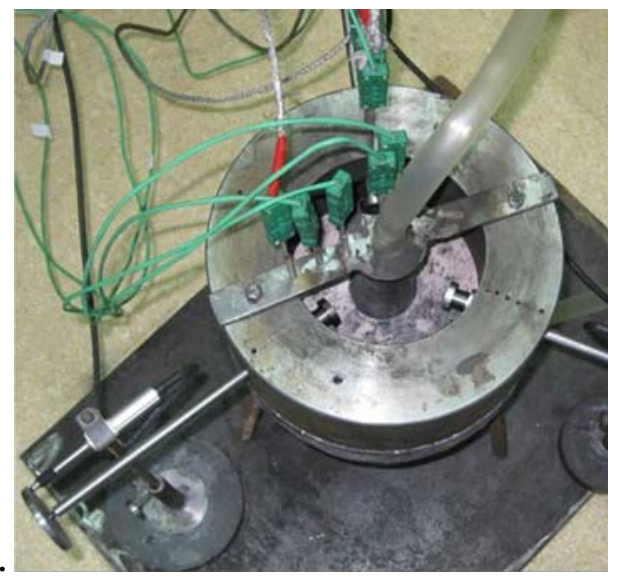

Fig. 1. Experimental set-up: a. schematic top view; b. Physical set-up.

The bottom of the mould is insulated with ceramics and, during pouring, solidification and cooling of the melt, the core is cooled by water at the room temperature. A schematic top view of the experimental set-up and the physical set-up are presented in Figure 1.

The melt material is $\mathrm{Al}-7 \% \mathrm{Si}$ (also known as $\mathrm{AC} 4 \mathrm{CH}$ ), a very used alloy in automotive industry for manufacturing of car wheels due to its very good castability.

After filling, the melt and mould thermal field dynamics was monitored with K-type thermocouples by a computerized system consisting in a programmable logic controller (PLC) connected to a Windows operation system. The experimental data acquisition module is presented in Figure 2. The data acquisition module has an analogue input module, which the K-type thermocouples used for measuring the temperature were connected to. To compensate the cold junction, a PT100 thermo-resistance was connected to the module. It is already known that a thermocouple measures the temperature difference between two points, and not the absolute temperature. To measure a temperature, one of the junctions - usually cold junction - is considered to be the known reference temperature. For compensation, an artificial cold junction was introduced. 


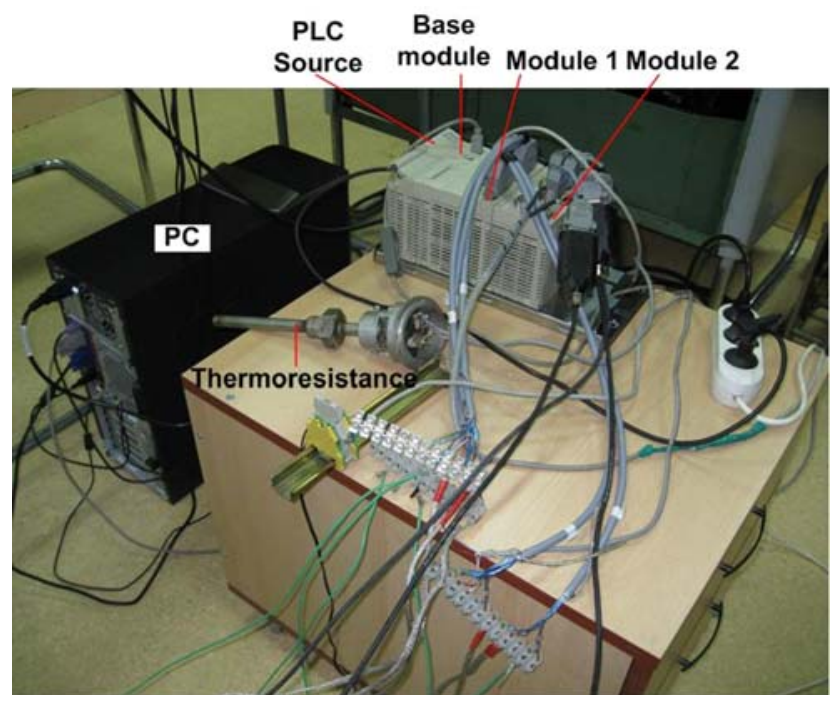

Fig. 2. Experimental data acquisi6tion module.

This cold junction uses a thermal sensitivity device in order to measure the input connection temperature, thus minimizing any temperature gradient between the terminals. Because of that, the voltage of a known cold junction can be simulated and applied the proper correction to. This technique is known as the compensation of the induced effect of the cold junction.

\subsection{Thermal field dynamics measurement}

The thermal field dynamics of the core, cast and mould was monitored by thermocouple measurement along radial direction. The mould temperature was monitored with thermocouples 1-3, the melt temperature was monitored with thermocouples 4-6 and the core temperature was monitored with thermocouple 7, as it is shown in Figure 1a. The experimental results are presented in Figure 3.

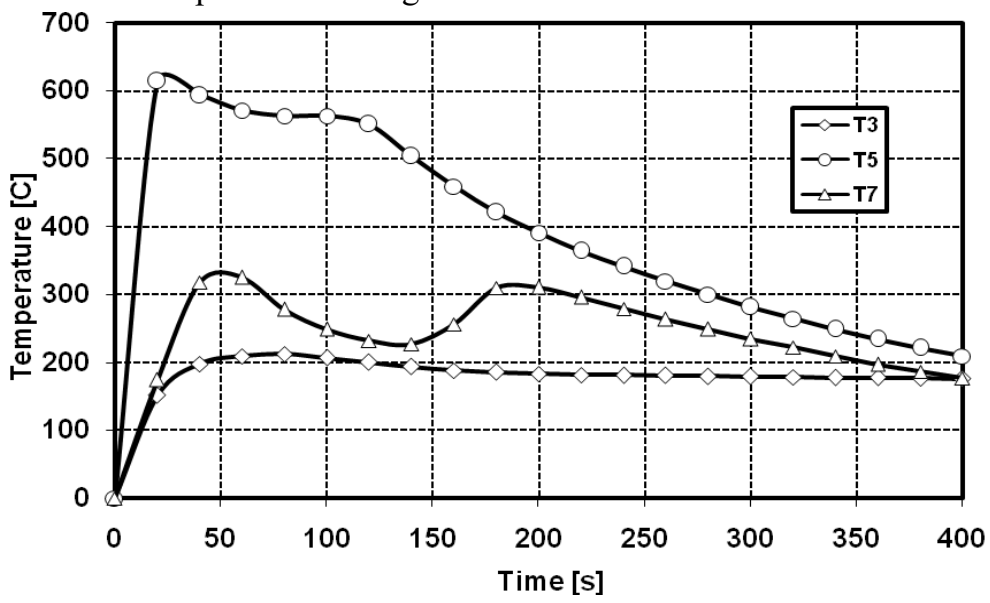

Fig. 3. Experimental thermal field dynamics in the core (T7), cast part (T5) and mould (T3).

The first dendrite skeletons of the microstructure cast material form when mould filling phase ends. Due to the fact that these dendrite skeletons are fragile, they are dissolving into 
the liquid because cannot withstand the metallostatic pressure of the melt. At the moment that dendrite skeletons become strong enough to be able to withstand the metallostatic pressure of the melt, the cast microstructure starts to form.

After this phase, the cast temperature decreases slow, being directly related to the capacity of mould and cooling water to take the heat from cast part and to transport this heat to ambient.

At the moment the first dendrite skeletons form, an early contact pressure occurs at the cast/core interface. When these primarily skeletons are dissolved by the mass of liquid, which is considerable larger, the core will have a temperature drop. At the time the new dendrite skeletons form, the melt will be again in strong contact with the core surface, this situation leading to the occurrence of contact pressure. The contact pressure, along with the surface roughness of the core represents the main factor which dictates the heat quantity to be absorbed by the cooling water.

At the beginning, the outer wall of the mould is in contact with the melt, but once the finale dendrite skeletons form, due to shrinkage, at the cast/mould interface an air gap occurs. This air gap plays a barrier role for the heat transfer due to its low conductivity. This is the reason that mould has a slight temperature drop after 150 seconds after filling phase ends.

Using the experimental data, heat transfer coefficients at the cast/core and cast/mould interfaces may be determined. The empirical methods used for heat transfer coefficients determination vary, but, generally, are grouped in direct methods and inverse methods.

\section{Artificial neural model and data prediction}

ANN is a very complex mathematical model which is used in many domains, such statistics, image processing, manufacturing or communications $[7,9]$.

The proposed neural model is composed of three layers, each of these layers having a certain number of neurons which operate in parallel and communicate with neurons from other layers. ANN model used for the prediction of mould thermal field dynamics is presented in Figure 4.

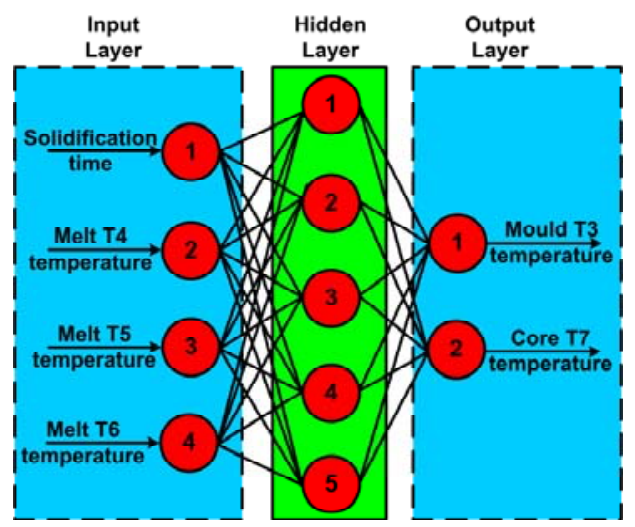

Fig. 4. Neural model used for mould temperature prediction.

The first layer of the neural model has 4 neurons: the time and melt temperature in three points, which was measured with thermocouples 4-6 (see also Figure 1a). The second layer is hidden layer and has 5 hidden neurons.

The last layer of the neural model has 2 neurons, the mould temperature (thermocouple 3 ) and core temperature (thermocouple 7), both measured very close to the interfaces with 
cast part. It is obviously that the thermal field dynamics measured in points 1 and 2 of the mould (Figure 1a) may be also output parameters.

For training, testing and validation of the proposed neural model, 130 data sets were used, as it follows: 71 data sets - for training, 10 data sets - for testing and 49 data sets for validation.

\subsection{Results and discussion}

At the beginning, the neural model was trained with 71 data sets, in order to create the mathematical model and to learn the network. Following each training, the mathematical model was tested with 10 data sets and the error for each data set was calculated.

When the average error is below 3\%, the next step is model validation. In the end, the average error of validation data sets must be also below 3\%. The comparative results of the experiment and prediction are shown in Figure 5.

The results presented in Figure 5 show a very good agreement with experimental data, the average error is $0.9 \%$ for mould temperature prediction and $2.47 \%$ for core temperature prediction, which lead to the validation of the neural model.

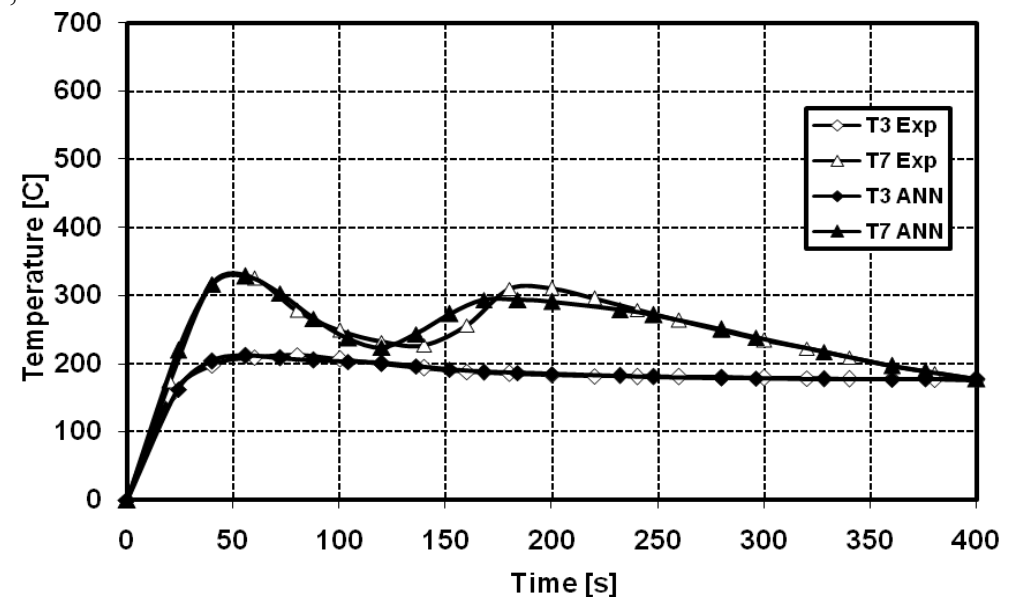

Fig. 5. Comparison between the experimental and predicted values of thermal field dynamics in the mould (T3) and core (T7).

Better results are obtained in case of core prediction due to the fact that the experimental temperature dynamics presents uniform evolution with no slopes. Higher values of error are between 140 and 160 seconds, after the cast parts microstructure is already formed (the solidification phase ends at about 120 seconds after mould filling), so this will not affect the cast part properties.

This neural model can be integrated within a neural control scheme for off-line or even on-line control of the casting process.

The novelty brought by this work consists in a new technique for casting process control. After setting up an experimental data base of the cast and mould thermal history, this can be used for generating a neural model. Then, the neural model can be off-line or on-line interrogated in order to interfere in real time, in solidification/cooling phase of the cast part, for controlling its microstructure and, as a consequence, to control the mechanical properties as strength.

The parts strength is very important if consider the dynamic stresses during their functioning. Knowing the mechanical properties level of the cast parts even from the 
designing stage brings great advantages, mainly due to the minimizing the experimental testing costs.

At the same time, based on already created neural model, there can be drawn up timetemperature-strength correlation diagrams, which can be used in designing and manufacturing stages of the cast parts. Moreover, depending on the material, a timetemperature-strength variation law can be determined.

Anyway, the presented neural model has some limitations: the prediction of the thermal field dynamics can be carried out for core and mould temperatures within the temperature range $\left(T_{\min } \ldots T_{\max }\right)$ measured by thermocouples in the cast part.

\section{Conclusions}

An ANN is a very complex mathematical model which may be used for prediction of process parameters in manufacturing engineering. The use of ANN significantly reduces the computing time of numerical simulation and the results are in very good agreement with physical experiment. The paper presents the prediction of thermal field dynamics of mould in casting process of a hollow cylinder cast part made of Al-7\% Si Alloy.

The average error is $0.9 \%$ for mould temperature prediction and $2.47 \%$ for core temperature prediction, which lead to the validation of the proposed neural model. These values are below an imposed value of $3 \%$ that will not affect the mechanical properties of the cast part.

The novelty of this work consists in an experimental data base of the cast and mould thermal history that can be used for generating a neural model, which can be off-line or online interrogated in order to modify in real time the thermal path of the solidifying alloy, for controlling its microstructure and, as a consequence, to control the mechanical properties of the final part, such as strength.

The model presented in this paper may be further used in conceiving and testing of a neural controlling set-up of the process, on-line or even off-line, in order to manufacture cast parts with improved mechanical properties.

This work was carried out after a mobility grant of the Romanian National Authority for Scientific Research and Innovation, CNCS - UEFISCDI, project number PN-III-P1-1.1-MC-2017-2211.

\section{References}

1. G. Palumbo, V. Piglionico, A. Piccininni, P. Guglielmi, D. Sorgente, L. Tricarico, Appl. Therm. Eng. 78, 682 (2015)

2. L. Kovacevic, P. Terek, A. Miletic, D. Kakas, Heat M. Transf. 50, 1115 (2014)

3. S. Bouhouche, M. Lahreche, J. Bast, A. Aut. Sin., 34, 701 (2008)

4. P. G. C. Manjunath, K. S. Arun, B. P. Mahesh, J. Manuf. P., 32, 199 (2018)

5. L. Zhang, L. Li, Heat M. Transf. 49, 1071 (2013)

6. F. Wang, K. Wu, X. Wang, Z. Han, Model. Cast. Solidif. Proc. MCSP 2017, 14, 327 (2017)

7. X. Yang, J. Zhu, Z. Nong, D. He, Z. Lai, Y. Liu, F. Liu, Trans. Nonfer. Met. Soc. China, 23, 788 (2013)

8. F. Susac, I. Constantin, M. Banu, A. Epureanu, F. Potecaşu, M. Marin, An. Dun. J. Univ. Galati, Fasc V, 91 (2011)

9. N. M. Vaxevanidis, J. D. Kechagias, N. A. Fountas, D. E. Manolakos, The O. Constr. Build. Tech. J., 8, 389 (2014) 